DISKUSSIONSFORUM

\section{Die hermeneutische Erweiterung der Technikfolgenabschätzung}

\author{
von Armin Grunwald, Institut für Technikfol- \\ genabschätzung und Systemanalyse (ITAS), \\ Karlsruhe
}

Technikfolgenabschätzung ist schon dem Namen nach konsequentialistisch ausgerichtet: Es geht um die Erforschung und Bewertung von Folgen. Damit dies möglich ist, müssen Technikfolgen also in gewisser, nicht beliebiger Weise mit Gründen antizipierbar sein. In diesem Beitrag gehe ich der Frage nach, ob Orientierung im Rahmen der TA auch möglich ist, wenn diese Voraussetzung nicht mehr erfüllt ist. Die These ist, dass Orientierung dann nicht mehr im Rahmen eines konsequentialistischen Zugangs, sondern nur eine hermeneutische Analyse der debattierten Zukünfte möglich ist.

\section{Der Konsequentialismus der Technikfolgenabschätzung}

Die systematische Befassung mit den Folgen der wissenschaftlich-technischen Entwicklung hat bereits eine jahrzehntelange Tradition. Sie hat Forschungs- und Reflexionsrichtungen hervorgebracht wie die Technikfolgenabschätzung (TA), die Risikoforschung, Wissenschafts- und Technikethik, das Feld der STS-Studies (science, technology \& society), die ELSI-Analysen (ethical, legal and social implications) und die EHSForschung (environment, health \& safety).

Während die spezifischen Ziele dieser Ansätze ebenso differieren wie ihre wissenschaftlichen Kontexte und die verwendeten Methoden, so besteht doch eine große konzeptionelle Übereinstimmung darin, dass es darum gehe, Technikfolgen $z u$ antizipieren. Hauptsächlicher Gegenstand der jeweiligen Analyse ist die Erforschung von Folgen, die es noch gar nicht gibt und vielleicht auch nie geben wird (Bechmann et al. 2007). Das Wissen über diese Folgen soll Orientierung für Gesellschaft und Politik geben, z. B. für Entscheidungsprozesse über Forschungsförderung bzw. Regulierung oder in der deliberativen Austragung von Technikkonflikten (Abb. 1)

Abb. 1 Das konsequentialistische Muster der Generierung von Orientierung durch Zukunftsbetrachtungen

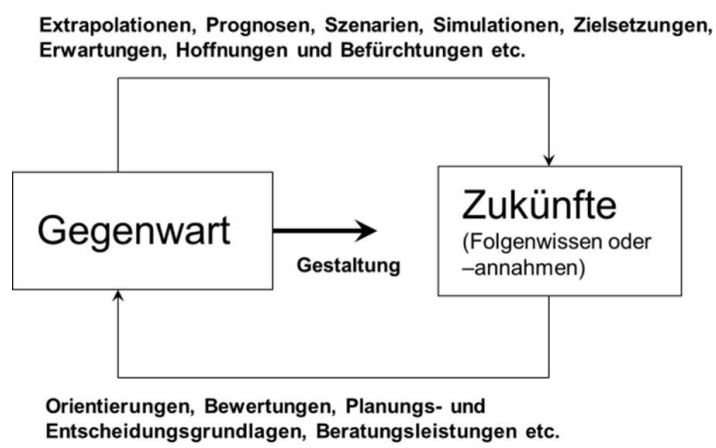

Quelle: Grunwald 2013a, modifiziert

Die Grenzen dieses konsequentialistischen Ansatzes werden vielfach gesehen und in den genannten Ansätzen der Folgenforschung reflektiert, gerade auch in der TA, in der der prognostische Ansatz ihrer frühen Phase sich rasch als nicht einlösbar erwies (Grunwald 2003). Eine wesentliche Reaktion auf diese Erkenntnis zeigt sich in der weitgehenden Verabschiedung prognostischer Erwartungen an die Technikfolgenforschung zugunsten eines Denkens in alternativen Zukünften, das seine methodische Fundierung vor allem in der Szenarientechnik hat. Auf diese Weise wurden technikdeterministische Vorstellungen überwunden, Gestaltungsmöglichkeiten konnten angesichts einer offenen Zukunft in den Blick genommen werden.

Freilich verbleibt auch dieser ,szenarische“ Ansatz im konsequentialistischen Muster. Es wird nur statt der einen prognostizierten Zukunft ein Bündel möglicher Zukünfte in den Blick genommen, z. B. in dem bekannten Muster von Business as Usual-, Worst Case- und Best Case-Szenarien, um ,robuste“ Handlungsstrategien entwickeln zu können. Unklar ist, was noch möglich ist, wenn eine sinnvolle Eingrenzung der Zukunft auf ein Szenarienbündel nicht mehr gelingt.

\section{Die Herausforderungen der NEST-Debatte}

Etwa seit dem Jahr 2000 wurden die TA und andere Ansätze der Technikfolgenreflexion vor die He- 
rausforderung der heute so genannten NEST (new and emerging sciences and technologies) gestellt. Dort zeigte sich rasch, dass der konsequentialistische Ansatz nicht funktioniert. Erste Hinweise finden sich in der Debatte zur Nanotechnologie (Zülsdorf et al. 2011). Angesichts des extremen Schwankens früherer Reflexionsansätze zwischen Paradieserwartungen und Befürchtungen der Apokalypse (Grunwald 2006) war hier nicht nur der prognostische Ansatz, sondern sogar das Denken in alternativen Zukünften illusorisch. Zu groß und unbestimmt erschien der Möglichkeitsraum des Zukünftigen, als dass hier noch eine vernünftige Eingrenzung auf einige Szenarien möglich gewesen wäre. Die Debatten zum Human Enhancement, zur Synthetischen Biologie und zum Climate Engineering führten auf ähnliche Probleme der Orientierungsleistung (Grunwald 2013b).

Konzeptionelle Reaktionen auf diese Situation ließen nicht auf sich warten. Der Vorschlag eines Vision Assessment (Ferrari et al. 2012), die Kritik an der spekulativen Nano-Ethik (Nordmann 2007), die Skizze einer explorativen Philosophie (Grunwald 2010) und verschiedene, meist verstreute Hinweise auf die hermeneutische Seite der Technikreflexion (z. B. Burg 2014; Torgersen 2013) sind hier zu nennen. Gemeinsam ist diesen Ansätzen eine nicht-konsequentialistische Perspektive. Denn wo das „Folgenwissen“ nur in epistemologisch nicht klassifizierbaren, spekulativen Zukunftserwartungen, Visionen oder Befürchtungen besteht, muss jeder Versuch misslingen, durch eine Folgenanalyse zur wissenschaftlichen, gesellschaftlichen oder politischen Orientierung beizutragen.

Die Frage ist dann, ob in einer solchen Situation, wenn jeglicher Anspruch auf Antizipation aufgegeben werden muss (Nordmann 2014), im Rahmen der TA oder anderer wissenschaftlicher Forschungs- und Reflexionsansätze überhaupt noch belastbare Orientierung geleistet werden kann. Vielleicht muss Wissenschaft aufgrund von nicht behebbarem Wissensmangel hier passen und den Gang der Entwicklung sich selbst überlassen. Dieser resignativen Einschätzung folge ich nicht. Stattdessen möchte ich hier die These entfalten (auf der Basis von Grunwald 2014b), dass Orientierung in Form von wissenschaftlicher Politikund Gesellschaftsberatung auch in diesen Fällen möglich ist - allerdings in anderer Form. Es bleibt die Option, die teils sehr lebhaften und kontroversen Debatten um NEST oder andere Wissenschafts- oder Technikfelder eben nicht als antizipatorische, prophetische oder quasi-prognostische Rede über Zukünftiges, sondern als Ausdruck unserer Gegenwart zu deuten. Nicht das, was in diesen Debatten mit mehr oder weniger Berechtigung über kommende Jahrzehnte gesagt wird, sondern was die Tatsache, dass diese Debatten heute stattfinden, über uns heute aussagt, wird zum Thema der Untersuchung und zur Quelle der Generierung von Orientierung gemacht. Mit diesem Perspektivenwechsel möchte ich eine hermeneutische Erweiterung der TA vornehmen.

\section{Modi der Orientierung durch Technikzukünfte}

Letztlich sind Technik(folgen)forschung und -reflexion problemorientiert, $d$. h. praktische Zwecke der Orientierungsleistung stehen, z. B. in der Politikberatung, im Vordergrund (Grunwald 2009). Die erbrachte Orientierung muss nachvollziehbar wissenschaftlichen Kriterien genügen, dies ist ihr Legitimationsgrund. Abhängig von der epistemischen Qualität des Folgenwissens sind unterschiedliche Typen der Orientierung möglich und kommen unterschiedliche Legitimationsstrategien für die Orientierungsleistung in Frage. Diese Beobachtung war Anlass für die folgende Dreiteilung (Grunwald 2014a; vgl. als Überblick die Tab. 1):

- mode 1 (prognostische) Orientierung: Durch die Prognose von zukünftigen Technologien und ihren Folgen soll ein belastbarer Rahmen erzeugt werden, um anstehende Entscheidungen, z. B. in Fragen der Regulierung oder des Ausbaus von Infrastrukturen, zu orientieren bzw. zu optimieren (z. B. Bullinger 1991). Dieser Ansatz wurde vor allem in der Anfangszeit der TA verfolgt und entspricht einem prognoseoptimistischen Vorgehen. Durch eine Reihe von Misserfolgen dieses Ansatzes und theoretischen Überlegungen, die grundsätzliche Schwierigkeiten aufgedeckt haben, wird dieser Ansatz zumindest in der TA gegenwärtig praktisch nicht mehr verfolgt (Grunwald 2003). 
- mode 2 (szenarische) Orientierung: An Stelle von Prognosen haben sich Szenarien als „mögliche Zukünfte“ in vielen Bereichen der TA, z. B. in Nachhaltigkeitsuntersuchungen, als Standardkonzept für das systematische Nachdenken über eine prinzipiell offene und daher nicht prognostizierbare Zukunft etabliert. Besonders häufig sind sie dort anzutreffen, wo ein Bedarf nach Orientierung zu komplexen Problemstellungen von übergreifender, gesellschaftlicher Bedeutung besteht und wo unterschiedliches Wissen, verschiedene Meinungen und Ansichten zu integrieren sind (z. B. Heinrichs et al. 2012). Ihre besondere Stärke entfalten sie dort, wo sich ,mögliche Zukünfte" von den weniger gut möglichen nachvollziehbar und transparent abgrenzen lassen, und wo in dem verbleibenden Raum der möglichen Zukünfte Unterscheidungen mit guter Orientierungsleistung eingezogen werden können (z. B. nach dem Muster von „,best case“" oder „worst case“ "Szenarien). Auf diese Weise hat TA gelernt, mit der Offenheit der Zukunft und der dadurch implizierten Nicht-Prognostizierbarkeit konstruktiv umzugehen und in dieser Offenheit Orientierungsmöglichkeiten $\mathrm{zu}$ entwickeln, z. B. durch robuste Handlungsstrategien.

- mode 3 (hermeneutische) Orientierung: Wenn das Zukunftswissen allerdings so unsicher ist oder die Zukunftsbilder so stark divergieren, dass es keine validen Argumente mehr für eine Abgrenzung zwischen „möglichen“ und „weniger oder gar nicht möglichen" Zukünften oder für eine Strukturierung in Form von Szenarien gibt, dann erscheinen Zukunftsprojektionen als beliebig und Orientierung auf dem konsequentialistischen Weg aussichtslos (s. o., insbesondere für das Feld der NEST). Es bleibt in dieser epistemologisch aussichtslosen Situation nur noch, im Sinne einer „hermeneutischen Wende“ (Grunwald 2014b) zu verstehen, warum das so ist, was diese Widersprüche über uns heute aussagen und was wir daraus für Orientierungszwecke lernen können.

Die Metapher des Zukunftskegels kann hier zur Illustration dienen, wenngleich die Suggestion eines festen Randes dieses Kegels problematisch ist. Die prognostische Orientierung entspräche einem Kegel mit dem Öffnungswinkel null Grad, also der Reduktion auf eine Zukunft. Die hermeneutische Orientierung antwortet auf die Situation, dass der Kegel, metaphorisch gesprochen, einen Öffnungswinkel von 180 Grad hätte und daher von sich aus nichts orientieren kann. Szenarische Orientierung deckte den Bereich dazwischen $a b$, und weil dieser Bereich vermutlich bei weitem der größte ist, hat auch dieser Typ der Orientierung die größte Bedeutung in der TA.

Tab. 1.: Wesentliche Eigenschaften der Modi der Orientierung durch Zukunftswissen

\begin{tabular}{|c|c|c|c|}
\hline & Prognostic & Scenario-based & Hermeneutic \\
\hline Approach to the future & one future & corridor of sensible futures & open space of futures \\
\hline Spectrum of futures & convergence as ideal & bounded diversity & unbounded divergence \\
\hline Preferred methodology & quantitative, model-based & $\begin{array}{l}\text { quantitative or qualitative; } \\
\text { participatory }\end{array}$ & narrative \\
\hline Knowledge used & $\begin{array}{l}\text { causal and statistical } \\
\text { knowledge }\end{array}$ & $\begin{array}{l}\text { models, knowledge of } \\
\text { stakeholders }\end{array}$ & $\begin{array}{l}\text { associative knowledge, } \\
\text { qualitative arguments }\end{array}$ \\
\hline Role of normative issues & Low & depends on case & High \\
\hline $\begin{array}{l}\text { Orientation } \\
\text { provided }\end{array}$ & $\begin{array}{l}\text { decision-making support, } \\
\text { optimization }\end{array}$ & robust action strategies & $\begin{array}{l}\text { self-reflection and } \\
\text { contemporary diagnostics }\end{array}$ \\
\hline
\end{tabular}

Quelle: Grunwald 2014a, modifiziert 


\section{Die hermeneutische Erweiterung der TA}

Um der Möglichkeit einer hermeneutischen Orientierung nachzuspüren, ist es erforderlich, die Technikzukünfte und deren Diversität und Divergenz in den Blick zu nehmen, die in den entsprechenden Debatten verhandelt werden und in der Regel „contested“ sind (Brown et al. 2000). Es muss darum gehen, ihre Ursachen und Quellen aufzudecken und nach ihrer „Bedeutung“ zu fragen, sowohl auf der Ebene einzelner Technikzukünfte wie auch auf der Ebene des Spektrums unterschiedlicher Technikzukünfte im gleichen Technikfeld. Diese Technikzukünfte sind soziale Konstrukte, erzeugt und ,hergestellt" durch Menschen, Gruppen und Organisationen zu je bestimmten Zeitpunkten (Grunwald 2006; Grunwald 2012). Zukunftsbilder entstehen aus einer Komposition von Zutaten in bestimmten Verfahren (z. B. den Methoden der Zukunftsforschung). Dabei gehen die je gegenwärtigen Wissensbestände, aber auch Zeitdiagnosen, Werte und andere Formen der Weltwahrnehmung in diese Zukunftsbilder ein. Die Divergenz der Zukünfte spiegelt die Pluralität der Gegenwart, und diese ist nicht nur eine Pluralität der Werte, sondern auch eine der wissenschaftlichen wie der außerwissenschaftlichen Meinungen über epistemisch nicht klassifizierbare Zukunftsbilder.

Damit erzählen Zukünfte, wenn prognostische oder szenarische Orientierungen nicht gelingen, ausschließlich etwas über uns heute. Technikzukünfte als ein Medium gesellschaftlicher Debatten (Grunwald 2012) bergen Wissen und Einschätzungen, die es zu explizieren lohnt, um eine transparentere demokratische Debatte und entsprechende Entscheidungsfindung zu erlauben. Die Erwartung an hermeneutische Orientierungsleistung besteht darin, aus Technikzukünften in ihrer Diversität etwas über uns, unsere gesellschaftlichen Praktiken, unterschwelligen Sorgen, impliziten Hoffnungen und Befürchtungen lernen zu können. Diese Form der Orientierung ist freilich weitaus bescheidener als die konsequentialistische Erwartung, mit Prognosen oder Szenarien „richtiges Handeln" mehr oder weniger direkt orientieren oder gar, wie es in manchen Verlautbarungen heißt, „optimieren“ zu können. Sie besteht letztlich nur darin, die Bedingungen dafür zu verbessern, dass demokratische Debatten und Zukunftsentscheidungen aufgeklärter, transparenter und offener ablaufen können.

Im Vergleich zum konsequentialistischen Paradigma mit seiner zentralen Ausrichtung auf Fragen der Art, welche Folgen neue Technologien haben können, wie wir diese beurteilen und ob und unter welchen Bedingungen wir diese Folgen willkommen heißen oder ablehnen, geraten in dieser Perspektive weitere Fragestellungen in den Blick (vgl. zu einigen Fragen auch Lösch/Müller 2014):

- Wie wird wissenschaftlich-technischen Entwicklungen, die ja zunächst im Labor nichts weiter als eben wissenschaftlich-technische Entwicklungen sind, eine gesellschaftliche, ethische, soziale, ökonomische, kulturelle etc. Bedeutung zugeschrieben? Welche Rollen spielen dabei z. B. (visionäre) Technikzukünfte? Wer schreibt diese Bedeutungen zu und warum?

- Wie werden Bedeutungszuweisungen kommuniziert und diskutiert? Welche Rollen spielen sie in den großen Technikdebatten unserer Zeit? Welche kommunikativen Formate und sprachlichen Mittel werden verwendet und warum? Welche außersprachlichen Mittel (z. B. Filme, Kunstwerke) spielen hier eine Rolle und was sagt ihre Nutzung aus?

- Warum thematisieren wir wissenschaftlichtechnische Entwicklungen in der jeweiligen Weise, mit den jeweils verwendeten Technikzukünften und mit den jeweiligen Bedeutungszuweisungen und nicht anders? Welche alternativen Bedeutungszuschreibungen wären denkbar und warum werden diese nicht aufgegriffen?

- Haben nicht auch traditionelle Formen der Technikfolgenreflexion (Prognostik, Szenarien) eine hermeneutische Seite? Werden vielleicht hermeneutisch bedeutsame Konstellationen hinter scheinobjektiven Zahlenreihen, Prognosen und in Diagrammen geradezu versteckt?

In der Beantwortung dieser Fragen erweitert sich das interdisziplinäre Spektrum der TA. Sprachwissenschaften, hermeneutische Ansätze in Philosophie und Geisteswissenschaften, Kulturwissenschaften und auch die Hermeneutik in der Kunst - insofern z. B. Technikzukünfte mit künstlerischen Mitteln erzeugt und kommuniziert werden - müssen an Bord der TA kommen. 
Es sollte hier nun nicht behaupten werden, dass die TA sich mit Fragen dieser Art noch nie befasst habe. TA als Diskursanalyse zum Beispiel war und ist hier teils nahe dran. Jedoch ist m. E. das Verhältnis zu anderen Formen der Orientierungsleistung durch TA bislang nicht systematisch geklärt worden, genauso wenig wie die hermeneutische Seite der TA einmal explizit in den Blick genommen wurde. Diese „hermeneutische Erweiterung" der TA sei damit zur Diskussion gestellt.

\section{Literatur}

Bechmann, G.; Decker, M.; Fiedeler, U. et al., 2007: TA in a Complex World. In: International Journal of Foresight and Innovation Policy 4 (2007), S. 4-21

Brown, J.; Rappert, B.; Webster, A. (Hg.), 2000: Contested Futures. A Sociology of Prospective Techno-Science. Burlington

Bullinger, H.-J., 1991: Technikfolgenabschätzung Wissenschaftlicher Anspruch und Wirklichkeit. In: Kornwachs, K. (Hg.): Reichweite und Potential der Technikfolgenabschätzung. Stuttgart, S. 103-114

Burg, S. van der, 2014: On the Hermeneutic Need for Future Anticipation. In: Journal of Responsible Innovation 1/1 (2014), S. 99-102

Ferrari, A.; Coenen, Chr.; Grunwald, A., 2012: Visions and Ethics in Current Discourse on Human Enhancement. In: Nanoethics 6/3 (2012), S. 215-229

Grunwald, A., 2003: Die Unterscheidung von Gestaltbarkeit und Nicht-Gestaltbarkeit der Technik. In: Grunwald, A. (Hg.): Technikgestaltung zwischen Wunsch und Wirklichkeit. Berlin, S. 19-38

Grunwald, A., 2006: Nanotechnologie als Chiffre der Zukunft. In: Nordmann, A.; Schummer, J.; Schwarz, A. (Hg.): Nanotechnologien im Kontext. Berlin, S. 49-80

Grunwald, A., 2009: Technology Assessment: Concepts and Methods. In: Meijers, A. (Hg.): Philosophy of Technology and Engineering Sciences 9. Amsterdam, S. 1103-1146

Grunwald, A., 2010: From Speculative Nanoethics to Explorative Philosophy of Nanotechnology. NanoEthics 4 (2), S. 91-101

Grunwald, A., 2012: Technikzukünfte als Medium von Zukunftsdebatten und Technikgestaltung. Karlsruhe

Grunwald, A., 2013a: Prognostik statt Prophezeiung - Wissenschaftliche Zukünfte für die Politikberatung. In: Weidner, D.; Willer, St. (Hg.): Prophetie und Prognostik. Verfügungen über Zukunft in Wissenschaften, Religionen und Künsten. München, S. 81-95
Grunwald, A., 2013b: Techno-visionary Sciences: Challenges to Policy Advice. In: Science, Technology and Innovation Studies 9/2 (2013), S. 21-38

Grunwald, A., 2014a: Modes of Orientation Provided by Futures Studies: Making Sense of Diversity and Divergence. In: European Journal of Futures Research 2/1 (2014), S. 1-9

Grunwald, A., 2014b: The Hermeneutic Side of Responsible Research and Innovation. In: Journal of Responsible Innovation 1/3 (2014), S. 274-291

Heinrichs, D.; Krellenberg, K.; Hansjürgens, B. et al. (Hg.), 2012: Risk Habitat Megacity. Heidelberg

Lösch, A.; Müller, M. (Hg.), 2014: Schwerpunkt „Risikodiskurse/Diskursrisiken: Sprachliche Formierungen von Technologierisiken und ihre Folgen". In: Technikfolgenabschätzung - Theorie und Praxis 23/2 (2014), S. 4-55

Nordmann, A., 2007: If and Then: A Critique of Speculative Nanoethics. In: NanoEthics 1/1 (2007), S. 31-46

Nordmann, A., 2014: Responsible Innovation. The Art and Craft of Future Anticipation. In: Journal of Responsible Innovation 1/1 (2014), S. 87-98

Torgersen, H., 2013: TA als hermeneutische Unternehmung. In: Technikfolgenabschätzung - Theorie und Praxis 22/2 (2013), S. 75-80

Zülsdorf, T.B.; Coenen, Chr.; Ferrari, A. et al. (Hg.), 2011: Quantum Engagements: Social Reflections of Nanoscience and Emerging Technologies. Heidelberg

\section{Kontakt}

Prof. Dr. Armin Grunwald

Institut für Technikfolgenabschätzung und

Systemanalyse (ITAS)

Karlsruher Institut für Technologie (KIT)

Karlstraße 11, 76133 Karlsruhe

Tel.: +49 721 608-22500

E-Mail: armin.grunwald@kit.edu

$\ll 》$ 